Case

Report

\title{
Transient Constrictive Pericarditis Following Cardiac Surgery
}

\author{
Koichi Maeda, MD, Shunsuke Saito, MD, Toru Toda, MD, Takayoshi Ueno, MD,
}

Toru Kuratani, MD, and Yoshiki Sawa, MD

\begin{abstract}
Transient constrictive pericarditis (CP) is quite rare, with only a few reports presented. Herein, we report 2 cases of transient CP following cardiac surgery. Case 1 was an 80-year-old man who underwent repair of a left ventricular free wall rupture, while Case 2 was a 69-year-old woman who underwent a conventional aortic valve implantation procedure. In both cases, constrictive features developed about 2 weeks after surgery, which were treated successfully by administrations of non-steroidal anti-inflammatory drugs (NSAIDs). Prior to performing a pericardiectomy, NSAID administration should be considered for affected patients who are hemodynamically stable.
\end{abstract}

Keywords: constrictive pericarditis, non-steroidal anti-inflammatory drug, cardiac surgery

\section{Introduction}

Constrictive pericarditis (CP) is considered to be a process of inflammation of the pericardium, which often leads to thickened, scarred, and calcified tissues that limits diastolic ventricular filling. Generally, a pericardiectomy is the only effective treatment for CP. However, a few reports have noted that $\mathrm{CP}$ was treated successfully by conservative therapy including administrations of non-steroidal anti-inflammatory drugs (NSAIDs) and steroids. $^{1-5)}$ Such a pathophysiology has been termed transient CP. Herein, we describe 2 cases of transient CP following cardiac surgery that recovered after receiving NSAID administrations.

Department of Cardiovascular Surgery, Osaka University Graduate School of Medicine, Suita, Osaka, Japan

Received: November 22, 2012; Accepted: July 31, 2013

Corresponding author: Yoshiki Sawa, MD. Department of Cardiovascular Surgery, Osaka University Graduate School of Medicine, 2-2 Yamadaoka, Suita, Osaka 565-0871, Japan

Email: sawa@surg1.med.osaka-u.ac.jp

(C)2014 The Editorial Committee of Annals of Thoracic and Cardiovascular Surgery. All rights reserved.

\section{Case Report}

\section{Case 1}

An 80-year-old man was referred to our department for a left ventricular free wall rupture (LVFWR) after acute myocardial infarction (AMI). Life-saving surgical repair of the LVFWR was performed on an emergency basis. The postoperative course was uneventful and the patient was discharged on postoperative day 9. On postoperative day 15 , he complained of dyspnea on effort and was readmitted. Chest $\mathrm{X}$ ray findings showed bilateral pleural effusion and a laboratory examination demonstrated mild inflammation (white blood cell count and C-reactive protein $8200 / \mathrm{mm}^{3}$ and $2.61 \mathrm{mg} / \mathrm{dl}$, respectively). Echocardiography revealed anomalous movement of the interventricular septum, known as "septal bounce," an adhesion between the pericardium and liver, and cyclic variation in the transmitral flow $\mathrm{E}$ wave velocity during respiration, typical signs of $\mathrm{CP}$ (Fig. 1A). With cardiac catheterization, a "dip and plateau" pattern was shown (Fig. 1B). Based on these findings, the patient was diagnosed with $\mathrm{CP}$ and administered an NSAID (aspirin, $2250 \mathrm{mg} /$ day) along with diuretic drugs, as the 

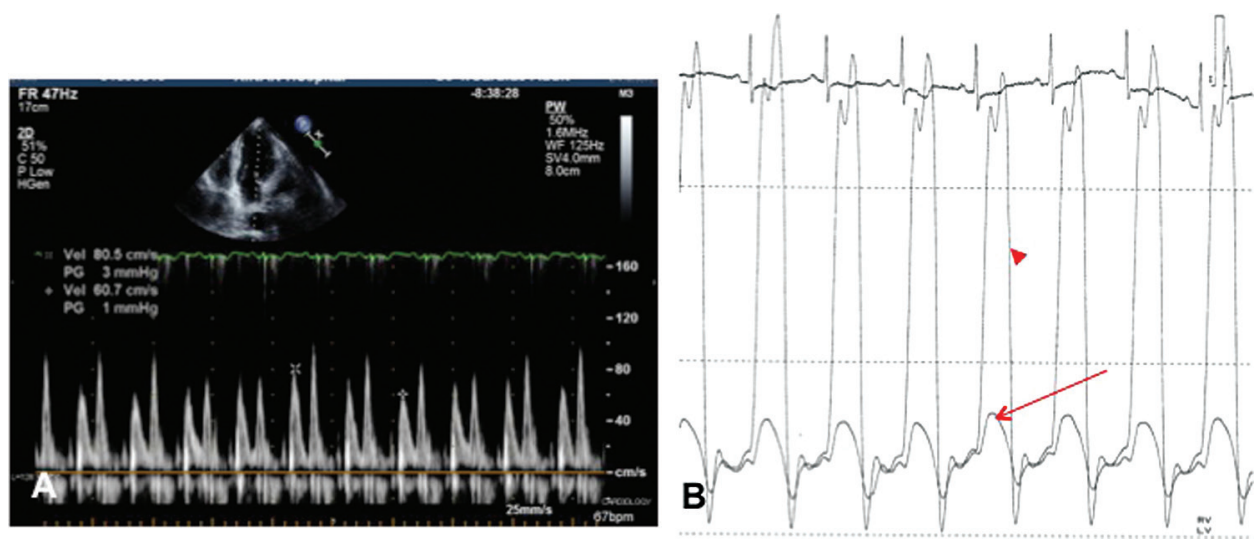

Fig. 1 (A) Cyclic variation in mitral inflow during respiration. A marked increase in the $\mathrm{E}$ wave of $32.6 \%$ with expiration was seen as compared with inspiration. (B) Cardiac catheterization revealed equalization of left (arrowhead) and right (arrow) ventricular diastolic pressures, which presented a dip and plateau pattern.

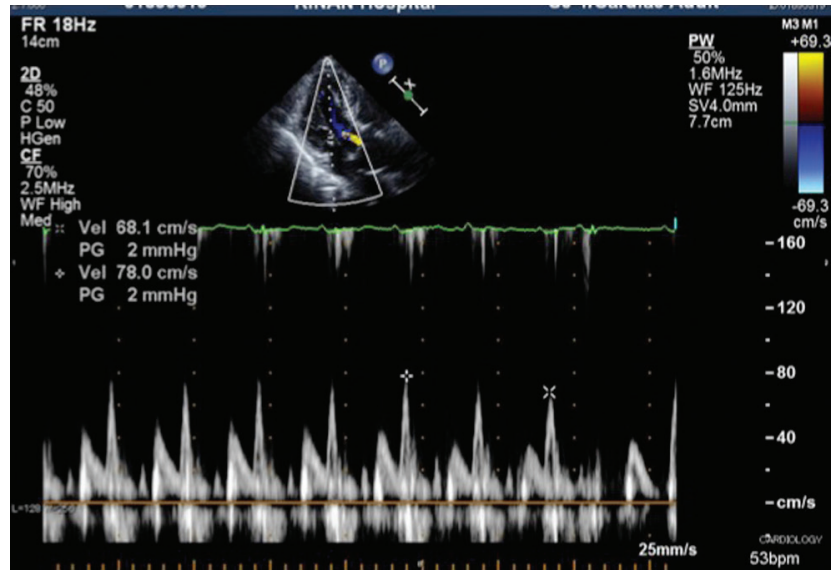

Fig. 2 Pre-discharge echocardiography showing that the cyclic variation of transmitral flow was significantly improved (from $32.6 \%$ to $14.5 \%$ ).

symptoms were not severe. Dyspnea disappeared completely on postoperative day 37 , while echocardiography showed significant improvements in septal bounce, adhesion of the pericardium, and cyclic variation of transmitral flow (Fig. 2). He was discharged on postoperative day 51 and has since undergone regular outpatient examinations without recurrence.

\section{Case 2}

A 69-year-old woman suffering from chest pain on effort was diagnosed with severe aortic stenosis and referred to our department for surgical treatment, during which a conventional aortic valve replacement procedure was performed. On postoperative day 14, the patient developed pretibial edema and right pleural effusion.

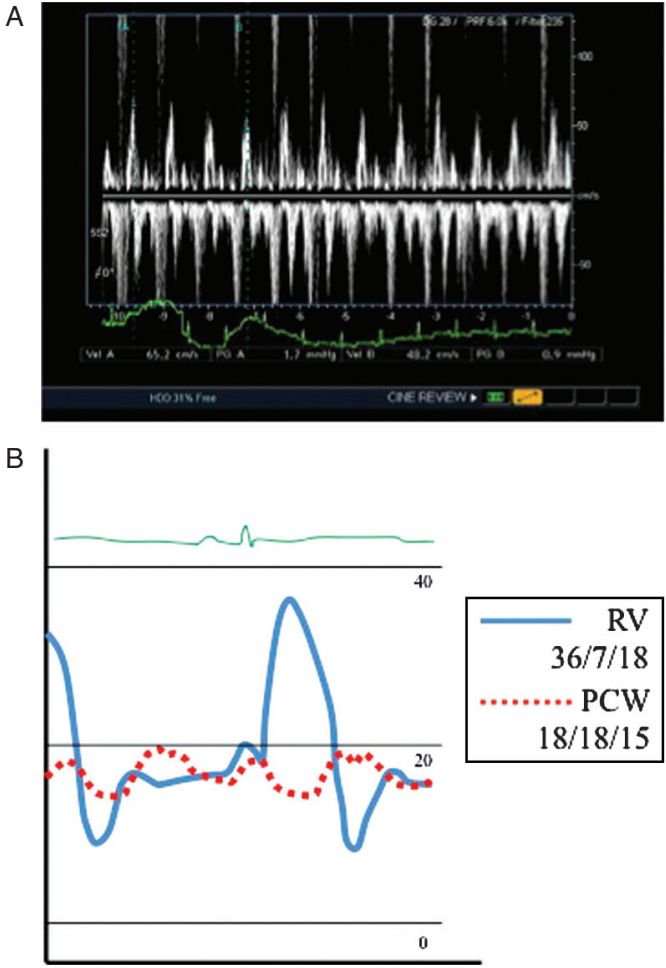

Fig. 3 (A) Cyclic variation in tricuspid inflow during respiration. A marked increase of $35.3 \%$ was seen with inspiration as compared with expiration. $\mathrm{E} / \mathrm{A}=1.77$. (B) Cardiac catheterization revealed a dip and plateau pattern. RV: right ventricule; PCW: pulmonary capillary wedge.

Echocardiography showed septal bounce, adhesion of the pericardium, and cyclic variation in tricuspid inflow during respiration (Fig. 3A). Furthermore, severe 


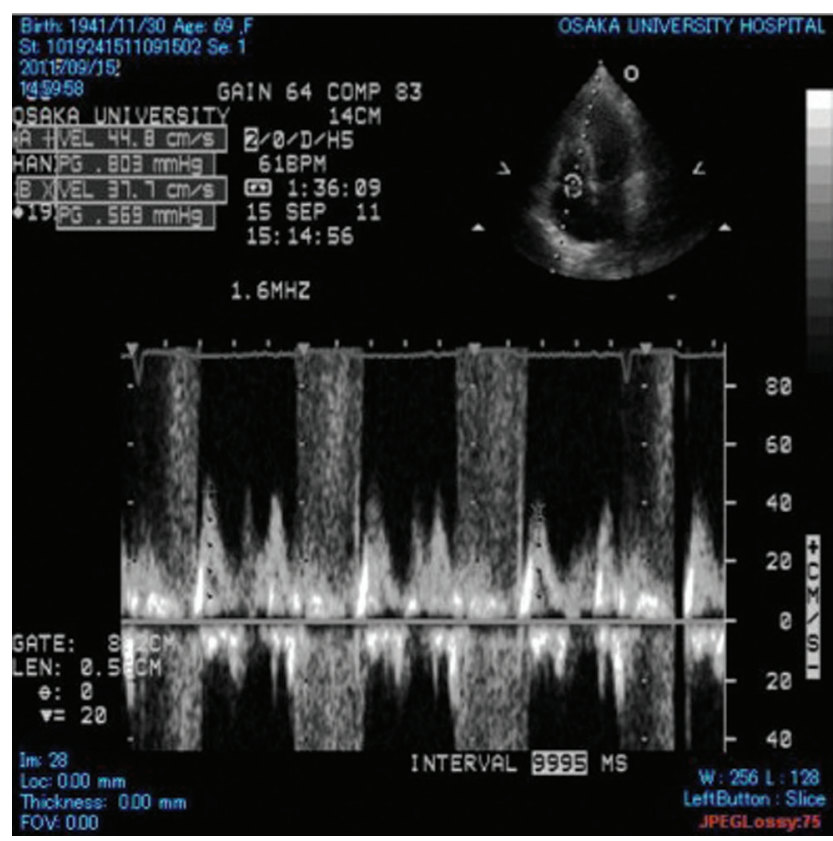

Fig. 4 Cyclic variation in tricuspid inflow was significantly improved from $35.3 \%$ to $18.8 \%$ in an outpatient echocardiography examination. $\mathrm{E} / \mathrm{A}=1.19$.

tricuspid regurgitation, which had not been recognized in preoperative echocardiography findings, was revealed. Cardiac catheterization demonstrated a dip and plateau pattern (Fig. 3B), and we made a diagnosis of CP. Considering the possibility of transient $\mathrm{CP}$, the patient was administered an NSAID (1500 mg/day), as she was hemodynamically stable. Following disappearance of pretibial edema and pleural effusion, she was discharged on postoperative day 83. An outpatient echocardiography examination on postoperative day 106 showed significant improvement in septal bounce, adhesion of the pericardium, and cyclic variation of transmitral flow (Fig. 4).

\section{Discussion}

CP following cardiac surgery is rare, with the incidence estimated to range from $0.2 \%-0.3 \%,{ }^{6}$ ) and only two cases (out of 1492 open heart surgeries from 2010 January to 2012 December: $0.13 \%$ ) were found in our institute. In affected patients, echocardiography can demonstrate septal bounce, adhesion of the pericardium, and cyclic variations in mitral and tricuspid inflow during respiration. In the inspiratory phase, an increase in venous return in the right atrium leads to a marked increase in right atrial pressure because of right ventric- ular diastolic dysfunction, which results in an elevated $\mathrm{E}$ wave in the trans-tricuspid valve as compared to in the expiratory phase. In the expiratory phase, left atrial pressure is higher than normal because of left ventricular diastolic dysfunction, which results in a marked increase in the trans-mitral valve pressure gradient and $\mathrm{E}$ wave as compared to in the inspiratory phase. In addition, cardiac catheterization demonstrates a dip and plateau pattern.

A pericardiectomy is the only known effective treatment for $\mathrm{CP}$, with a number of reports presented..$^{7-11)}$ Although an early pericardiectomy procedure has been advocated to improve results, operative and late mortality rates are not low. ${ }^{7)}$ In our Case 1, the operative mortality related to a pericardiectomy procedure might have been higher than usual because of post-myocardial infarction. Furthermore, Gongora, et al., reported moderate or severe tricuspid regurgitation such as seen in the present Case 2 is associated with increased mortality. ${ }^{10)}$

On the other hand, few cases of transient $\mathrm{CP}$ have been reported. ${ }^{1-5)}$ Sagrista-Sauleda and colleagues reported that 16 of 177 patients with acute idiopathic constrictive pericarditis had a transient course. ${ }^{1)}$ Also, Haley and colleagues investigated the cause and natural history of transient $\mathrm{CP}$, and reported that 36 of 212 patients (including 9 of 48 following cardiovascular surgery) who had echocardiographic findings of CP showed resolution of constrictive hemodynamics without a pericardiectomy in follow-up echocardiogram findings. ${ }^{2)}$ In that study, patients with transient $\mathrm{CP}$ were treated conservatively with NSAIDs, steroids, or others, with an average of 17.4 weeks needed to resolve transient $\mathrm{CP}$ in those that had undergone cardiovascular surgery. In the present cases, resolution was seen in 8 and 15 weeks, respectively. However, their report was a review of an echocardiogram database, thus there might have been more cases of transient $\mathrm{CP}$ for which a pericardiectomy was performed. Furthermore, there are no reports regarding how NSAIDs or steroids function in repair of $\mathrm{CP}$, though it has been suggested that patients who have constrictive features early in the course of illness and are hemodynamically stable should be considered for conservative therapy with NSAID administration before a pericardiectomy is pursued.

\section{Conclusions}

In summary, we report 2 cases of transient CP following cardiac surgery, which were successfully treated with administrations of NSAIDs. Patients who have 
constrictive features early in the course of illness and are hemodynamically stable should be considered for NSAID administration before a pericardiectomy is pursued. Nevertheless, there are few reports of transient CP, thus further investigation is mandatory.

\section{Disclosure Statement}

The authors declare that they have no competing interests.

\section{References}

1) Sagristà-Sauleda J, Permanyer-Miralda G, CandellRiera J, et al. Transient cardiac constriction: an unrecognized pattern of evolution in effusive acute idiopathic pericarditis. Am J Cardiol 1987; 59: 961-6.

2) Haley JH, Tajik AJ, Danielson GK, et al. Transient constrictive pericarditis: causes and natural history. J Am Coll Cardiol 2004; 43: 271-5.

3) Samara A, Bergman M, Vitrai J, et al. Recovery of transient pericardial constriction following steroid administration. Heart Lung Circ 2010; 19: 470-2.

4) Mercé J, Ramírez-Montesinos R, Sans-Roselló J, et al. Transient cardiac constriction with severe heart failure. Rev Esp Cardiol 2008; 61: 985-7.

5) Nakao K, Noguchi T, Kim J, et al. Transient constrictive pericarditis diagnosed by cardiac magnetic resonance, $67 \mathrm{Ga}$ scintigraphy, and positron emission tomography. Int J Cardiol 2009; 137: e70-2.

6) Cimino JJ, Kogan AD. Constrictive pericarditis after cardiac surgery: report of three cases and review of the literature. Am Heart J 1989; 118: 1292-301.

7) Chowdhury UK, Subramaniam GK, Kumar AS, et al. Pericardiectomy for constrictive pericarditis: a clinical, echocardiographic, and hemodynamic evaluation of two surgical techniques. Ann Thorac Surg 2006; 81: 522-9.

8) Ha JW, Oh JK, Schaff HV, et al. Impact of left ventricular function on immediate and long-term outcomes after pericardiectomy in constrictive pericarditis. J Thorac Cardiovasc Surg 2008; 136: 1136-41.

9) Tirilomis T, Unverdorben S, von der Emde J. Pericardectomy for chronic constrictive pericarditis: risks and outcome. Eur J Cardiothorac Surg 1994; 8: 487-92.

10) Góngora E, Dearani JA, Orszulak TA, et al. Tricuspid regurgitation in patients undergoing pericardiectomy for constrictive pericarditis. Ann Thorac Surg 2008; 85: 163-70; discussion 170-1.

11) Anderson CA, Rodriguez E, Shammas RL, et al. Early constrictive epicarditis after coronary artery bypass surgery. Ann Thorac Surg 2009; 87: 642-3. 